[CONTRIbUtion From the Research Laboratory of Appited ChEmistry, MASSAChUSETTS Institute of TeChNOLOGY, No. 31]

\title{
APPLICATION OF THE OCTET THEORY TO SINGLE-RING AROMATIC COMPOUNDS
}

\author{
BY ERNEST C. CROCKER \\ Received January 24, 1921
}

\section{Benzene}

Introduction.-The "octet" or "L,ewis-Langmuir" theory ${ }^{1}$ of atomic structure has done much to clarify our views concerning inorganic compounds in general. It has been applied with conspicuous success to some of the organic compounds of nitrogen, ${ }^{2}$ to some of the simpler hydrocarbons, and more recently, to addition compounds of ketones, ${ }^{3}$ yet up to the present, it has not been applied to the perplexing problem of the structure of aromatic compounds.

In the first stages of the evo'ution of this theory, octets were considered as necessarily cubical for, offhand, the cube appeared to be the form that must be the most stable one for 8 mutually-repelling electrons. This cubical octet, as a first approximation, was of great value, for it plainly indicated two different types of combination: the shared pair of electrons or non-polar "bond," and the electrostatic attraction between complete, charged octets, or octets and hydrogen kernels, which we recognize as ionic combination. It also made clear that "pentavalent" nitrogen does not have 5 organic-type bonds. No contributions of value, beyond those of the old valence theory, were recognized at the time as affecting carbon, hydrogen and oxygen as they exist in organic compounds. Moreover, the cubical form of carbon was not compatible with optical activity, which demanded atoms essentially tetrahedral.

As Lewis has recently stated, ${ }^{4}$ his first paper had not yet been published before he had decided that the octet was perhaps more likely tetrahedral than cubical. This tetrahedral type, however, demanded that electrons exist more or less in pairs, an arrangement apparently not consistent with their being electrically charged. The magneton theory of Parson, ${ }^{5}$ wherein he considered electrons as unit magnetic systems as well as units of negative electricity, however, suggested that perhaps this apparent pairing of electrons might be by virtue of the attraction of the magnetic field opposing the purely electrostatic property of repulsion. At any rate, the octet

${ }^{1}$ Lewis, Thrs Journal, 38, 762 (1916). Langmuir, ibid., 41, 868 (1919). Langmuir, ibid., 41, 1543 (1919). Langmuir, Science, 54, 59 (1921).

2 Langmuir, 'This Journal, 42, 274 (1920). .

${ }^{3}$ Conant, ibid., 43, 1705 (1921).

4 Lewis, Chem. Met. Eng., 24, 871 (1921).

'Parson, Smithsonian Inst. Pub., Misc. Coll., 65, No. 11 (1915). 
existing as 4 pairs of electrons, the pairs arranged as are the points of a tetrahedron, rather than as 8 electrons existing as are the corners of a cube, gives a form highly suitable for general organic chemistry.

In this paper, the pairing tendency of electrons will be kept constantly in mind. This accords with the present stage in the evolution of the octet theory. In accepting this tetrahedral form as perhaps the general form of the octet, and in emphasizing, the pairing tendency of the electrons, it is not necessary to abandon the advantages of the cube as a means of representation of octets, of which Lewis, Langmuir and others have made such excellent use. Also it is not inconsistent with octets being truly cubical in certain salt crystals, and possibly under other conditions where there are particular types of strain.

It is here proposed to consider some of the older, more or less accepted formulas for phases of the behavior of benzene, in the light of the octet theory, to develop from them a structure for benzene, and compare the properties which should result from such a structure, with those which are known to exist. This structure will then be applied, with slight modification, to other 1-ring aromatic compounds.

In the figures, a 1-plane type of representation, based on cubical octets, will be used. Squares in contact, corner to corner, represents a bond, or pair of shared electrons, and squares in side to side contact represents a double bond. Hydrogens are represented by $\mathrm{H}$ 's, and single electrons by dots.

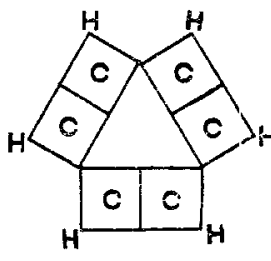

A.

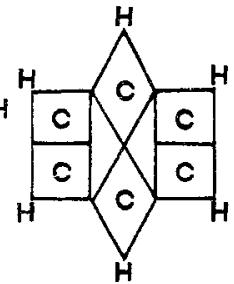

$B$.

Fig. 1.

$A$-Structure of benzene, according to Kekulé:<smiles>c1ccccc1</smiles>

$B$-Structure of benzene, according to Dewar:<smiles>C1=CC2C=CC1C2</smiles>

Octet Formulas for Benzene.-Fig. 1 shows the octet representation of 2 ring-type formulas for benzene, the static form of the familiar Kekule formula with alternate single and double bonds, and Dewar's formula with double bonds between Carbons 2 and 3 , and 5 and 6 , and bonds across the ring from carbons 1 to 4 . 
Fig. 2 is as close a representation as possible of the Baeyer "centric" formula. In this case, parts of the carbon sheaths are represented by broken lines, for the inside corners are single electrons, shown as dots. Such a formula is a violation of the octet principle, for each kernel is surrounded by only 7 electrons (or by 9 if the 2 adjacent ones are considered as shared). Moreover, the 6 electrons so relatively close together

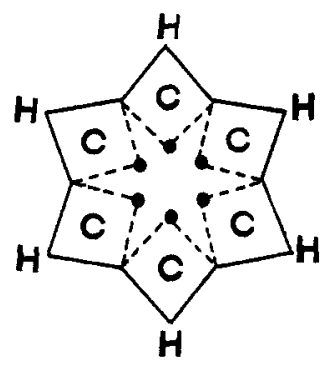

Fig. 2.-Baeyer's "Centric" formula for benzene:

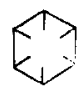

in the center of the ring with no positive kernel to hold them, should repel each other with considerable force.

If, however, the assumption is made that these 6 extra electrons are repelled to the outside of the ring of carbon-to-carbon junctions as depicted in Fig. 3, a much more reasonable structure results.

Each carbon now shares a pair of electrons with its neighbor on either side, and a pair with hydrogen. Besides these pairs, each carbon shares 1 "aromatic" electron with each adjacent carbon, making 8 electrons in

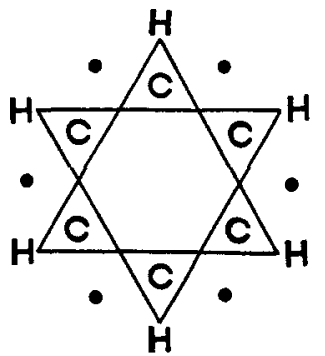

Fig. 3,-Benzene: all. Each hydrogen has 2 electrons, as usual. If the photograph of a model (Fig. 4) be examined, it will be noted that one face of each carbon octet consists of 4 electrons, as a diamond (a slightly distorted square) at the height of the hydrogen junctions. This is virtually two square faces turned $45^{\circ}$ with regard to each other. ${ }^{\circ}$

While it is not the purpose of this paper to discuss at length the physical properties of this arrangement, it may be mentioned that, reasoning from analogy with the case of helium, it seems highly probable that the six outside or "aromatic" electrons are continually vibrating in the plane of the ring, alternately pairing up momentarily with first one and then the other of the 2

' Since the submission of this paper to Thrs Journal, an article by Sir J. J. Thomson [Phil. Mag., 41, $510(1921)]$ treats of the shape of octets. He arrived at a general shape identical with the one here devised for benzene, and in particular pointed out that it might be applied to the "well-worn" case of benzene. 
adjacent aromatic electrons. The net physical effect of this oscillation would be that of 3 electrons moving around continuously in each direction in the plane of the ring, thus serving to bind together all the carbon atoms present into virtually a single atom. The dots, therefore, represent merely the average positions of these electrons.

Having derived a benzene ring which apparently satisfies octet requirements, let us consider what its properties should be, and how well they correspond with those known to exist for benzene.

First, such a figure is highly symmetrical, having 7 planes of symmetry as compared with 9 for a cube. Such a compound, then, would be expected to be highly stable - not easily attacked 'at any point-hence of relatively slight chemical reactivity. It contains no double bonds and so should be "saturated" to all save powerful addition agents. Any addition whatsoever should, moreover, break down the entire "aromatic" electronic structure, so that the remainder of the ring would be unsaturated (adopting a Kekule type of structure) and reactive for more addition.

The chemical properties of benzene

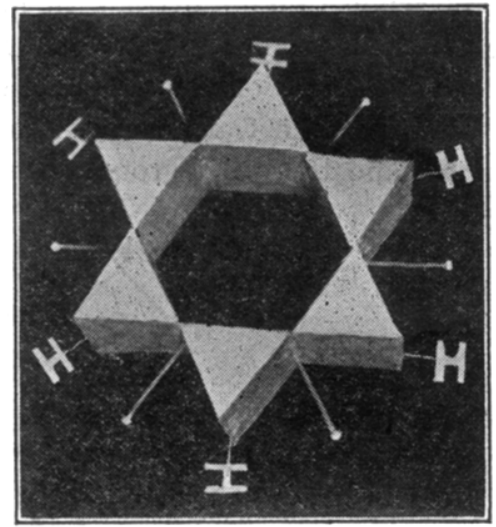

Fig. 4.

Photograph of model of benzene: are in entire accord with the above requirements. It is a stable compound, with "saturated" properties. In fact, hydrogens may be substituted by other groups without ethylenic addition taking place. Also, when a powerful addition agent such as ozone does succeed in combining, it never forms a mono-ozonide, but only a tri-ozonide. ${ }^{7}$ Chlorine and bromine cannot combine with it in the ethylenic-addition sense, and hydrogen combines only in the presence of carriers.

Substitution Behavior.-All positions in the above ring are equal, and only one kind of each disubstitution product should exist. This is in accord with the known number of derivatives of benzene.

It is to be expected that if some other element or radical replaces hydrogen at one carbon, the average positions of the "aromatic" electrons will be different from those in plain benzene. In general, if the substituent attracts the adjacent aromatic electrons more than did the replaced hydrogen, the electrons should move nearer to it than they did to the hydrogen; and conversely, groups with less attraction should not pull the aromatic electrons as far over as did the replaced hydrogen.

${ }^{7}$ Harries, Ann., 343, 311 (1905). 
If any one aromatic electron moves from its position midway between the carbons, the electrical equilibrium of the system is disturbed, and the others must move in such a manner as to restore it. Any movement of this kind must be in such direction that not only is the electrical equilibrium reëstablished, but also the pairing properties of the electrons must be satisfied. This means that if the electrons nearest to Carbon 1 are attracted towards it more than they were in benzene, the 4 remaining electrons will group around Carbons 3 and 5 . Likewise, if the substituents on Carbon 1 do not attract the nearby electrons as much as did the hydrogen kernel originally there, the groupings would then be around Carbons 2, 4 and 6. For convenience, the former grouping around Carbons 1 , 3 and 5 (when Carbon 1 is substituted) will be referred to as "Pattern o. p." and the other grouping as "Pattern $m . "$

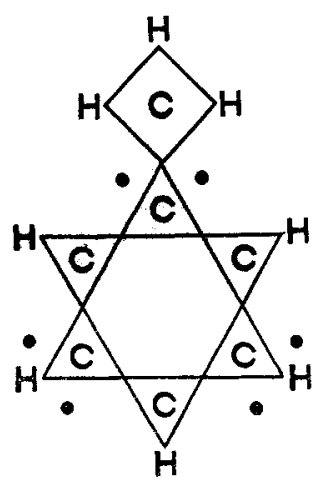

$A$.

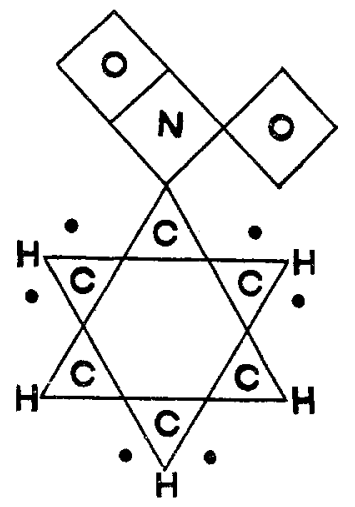

$B$.

Fig. 5.

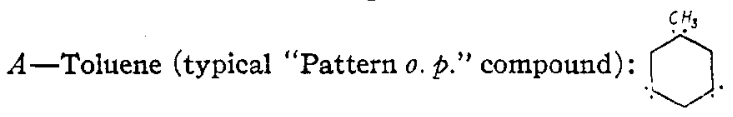

Note: The three hydrogens of the methyl group may conceivably be more nearly at equal distances from the juction with the benzene ring than is shown in the above somewhat symbolic, figure.

$B-$ Nitrobenzene (typical "Pattern $m$. " compound):<smiles>[C]1CCCCC1</smiles>

Note: The group $\mathrm{NO}_{2}$ in the octet system is depicted with one double-bonded, and one single-bonded oxygen.

The Two Benzene Patterns.-When an octet of large "effective" kernel charge such as chlorine (7) is attached to Carbon 1, the attraction on the aromatic electrons next to Position 1, will be greater than that of the hydrogen which was replaced, and Pattern $o$. p. will result. ("Effective" kernel charge is the true nuclear positive charge, minus as many units of charge as there are electrons inside the outer sheath. The ef- 
fective charge of hydrogen is 1 , of carbon 4 , of nitrogen 5 , oxygen or sulfur 6 , and the halogens 7 .) The side electrons of the chlorine octet have repulsive action on the aromatic electrons, yet not sufficient to neutralize the strong attractive action of the kernel. Oxygen with a kernel charge of 6 should also be expected to overcome the side repulsion and exceed the attractive power of a simple hydrogen kernel, so that hydroxyl and methoxyl would likewise cause Fattern $o . p$. When, however, the substituent is methyl, the carbon charge is small indeed (4), but there are hydrogen kernels at the octet sides, which partially reduce the repulsion there, due to the electrons, so that the same Pattern $o$. p. should exist in toluene (Fig. 5A). The group $\mathrm{NH}_{2}$, being midway between hydroxyl and methyl, should also cause the formation of Pattern o. p.

In Pattern o.p. the displacement of the aromatic electrons is such as to increase their attractifle force on the hydrogens of Carbons 3 and 5 , and decrease that on Positions 2, 4 and 6 . Hence Positions 3 and 5 are virtually "closed" so far as substitution is concerned, while Positions 2, 4 and 6 are "open" with the hydrogens much more lightly held. If substitution occurs in such compounds as chlorobenzene, phenol, aniline and toluene, one would expect it to be ortho-para, with not more than traces of meta products. Actually, the ortho-para compounds are formed, almost exclusively. ${ }^{8}$

All of the above-mentioned typical substituents which give Pattern o. $p$. are characterized by having a total attractive force (effective kernel charge plus hydrogen kernels on the sides of the octet next to the aromatic electrons) of not less than 6 , compared with repulsive charges of only 4 (the negative electrons on the adjacent sides). There is another group of substituents, however, including; the radicals $\mathrm{COOH}, \mathrm{CHO}, \mathrm{CN}$ and $\mathrm{NO}_{2}$, which have the same 4 repulsive charges, and only 4 or 5 total attractive charges by the above definition. As a result it would be expected that these groups would attract the aromativ electrons less than the adjacent hydrogens of the rings, and Pattern $m$ would result, leaving only the meta hydrogens open for ready substitution. This prediction from purely structural considerations is again verified by fact.

There is a large number of more complicated substituents with extra octets spreading out over the adjacent aromatic electrons, the net effects of which are more difficult to calculate in view of our lack of accurate knowledge as to the comparative sizes of the atoms and the distances involved. Considering the most important of these with some care, the conclusion has been reached that the type of substitution products given in these cases is frequently definitely predictable, and is at the most not in any way inconsistent with the foregoing considerations. The main

\& Hollemann, "Substituenten in den Benzolkern," Leipzig, 1910, Schwalbe, "Benzol Tabellen," Berlin, 1903. 
basis of the theory at the present time must, however, rest on its clean-cut predictions with regard to the simpler substituents as above discussed.

Some Polarity Considerations.-While the foregoing discussion explains in a very satisfactory manner the most important and mysterious property of aromatic compounds, it is recognized that there is, in addition, some superimposed factor which tends to make substitution much easier in certain cases than in others, and to favor para rather than ortho, or vice versa, when the above theory would indicate that they were equally open to substitution. The writer believes that these last-mentioned variations do not in any sense modify the validity of the foregoing discussion but are due to an additional or superimposed force, namely, the electrical polarity of the molecule.

Any molecule, taken as a whole, is electrically neutral; that is, it contains an equal amount of positive and negative electricity. The distribution of the negative charges (sheath electrons) is essentially uniform, and probably strictly so in compounds containing only carbon, nitrogen, and oxygen octets (which are at least approximately of the same size), so need not be further considered in this connection. The positive or kernel charges, however, are not so uniformly distributed. Octet kernels may vary from 4 to 7 in magnitude of effective positive charge and some charges may exist as hydrogen kernels outside of octets.

Now, a distributed charge will always have a smaller effect at a distance than will the same charge concentrated, because the attractive effect of electric charges adds vectorially and not arithmetically. Thus, in a compound such as methyl chloride, the chlorine end will act on any electrical system outside the molecule as though it were a positive pole and the methyl end as though it were a negative pole. Such a compound may be compared with a bar magnet.

When a bar magnet is touched to a mass of iron, the pole which was at the point of contact appears at the far end of the iron so that the composite mass has but one pair of poles-at its opposite ends. Similarly, if chlorine is attached to a benzene ring, it would be expected that a negative pole would appear at the far end of the ring and if a methyl group is attached to a benzene ring, it would be expected that a positive pole would appear at the far end (this effect being produced probably by a slight shifting of all the positive and negative charges in the molecule, as suggested by Lewis, in 1916).

If the far end of the ring (para, and to less degree, meta positions) is negative in polarity, as in chlorobenzene or nitrobenzene, the positive hydrogen kernels in those positions will be held more firmly than normally, and it is to be expected that substitution in these positions would be more difficult than usual. Likewise, with a positive pole at the far end of the ring, as in toluene or aniline, the para hydrogens will be held more loosely, 
thus making substitution easier for any kind of entering substituent: chlorine, nitro or diazo radicals. It would, therefore, be expected that for a given benzene pattern, those radicals such as chlorine or carboxyl which cause a negative pole at the far end (commonly termed "negative" radicals) will make substitution more difficult in the para position than, for instance, methyl or amino radicals. Also, it is to be expected that Pattern $m$. compounds such as benzoic acid and nitrobenzene, having, as they all do, strongly negative groups at the "top," should substitute with more difficulty than Pattern o. p. rings with positive or weakly negative groups. The facts are entirely in agreement with these deductions.

The ortho positions, being about midway between the two poles of a mono-substituted benzene, would be comparatively little affected as to ease of substitution by the polarity of the ring as a whole, but might be affected by the local action of the radicals which form Pattern o.p., this local action being perhaps the largest factor in "steric hindrance." In comparing the ease of substitution in para and ortho positions, it must be remembered that there are two oriho positions to one para available.

The foregoing polarity consideration is to be noted as the general effect of groups, such as will be observed when the distance between the ends of the molecule is considerable. This applies in large measure to groups situated on opposite sides of a benzene ring. There is another condition, however, and that is the local action of a polar group; the influence it has which is a direct result of its own action, and which extends to the distance of one or more atoms from the group.

A highly concentrated nuclear sharge ( 7 ), as in chlorine, or group of concentrated charges (17), as in the nitro group has a strong local action (much more extensive for the larger group $\mathrm{NO}_{2}$ than for $\mathrm{Cl}$ ). The effect on hydrogen nuclei for one or more atoms distance from the polar group would be repulsion, which should appear as ionization. This local action should fall off rapidly with distance; thus the compound, HC thas strong ionization and the compound, $\mathrm{HOCl}$, comparatively slight ionization.

"Negative" and "positive" groups as many organic chemists understand the terms may be defined from a consideration of the local effects. Those groups which make hydroxyl more acid than does hydrogen in the compound $\mathrm{H}-\mathrm{OH}$ are negative. Thus $\mathrm{SO}_{3} \mathrm{H}-\mathrm{OH}$ and $\mathrm{NO}_{2}-\mathrm{OH}$ are more acidic than is $\mathrm{H}-\mathrm{OH}$, making these groups negative. On the other hand, $\mathrm{CH}_{3}-\mathrm{OH}$ or $\mathrm{NH}_{2}-\mathrm{OH}$ are more basic than $\mathrm{H}-\mathrm{OH}$, in that the $\mathrm{H}$ of the $\mathrm{OH}$ group is less acidic than in water. In other words, a group is negative which, when joined to $\mathrm{OH}$, allows the $\mathrm{H}$ of the $\mathrm{OH}$ to split off; whereas it is positive if it allows the whole group $\mathrm{OH}$ to split off. $\quad(\mathrm{OH}$, joined to a weakly positive group, as in $\mathrm{CH}_{\mathrm{i}} \mathrm{OH}$, can in addition have the $\mathrm{H}$ of the $\mathrm{OH}$ replaced by more positive radicals such as $\mathrm{Na}$ and $\mathrm{K}$.)

By this definition, $\mathrm{OH}$ is a weakly negative group, since the compound 
$\mathrm{HO}-\mathrm{OH}$ has feeble acid properties, yet its feeble negative tendencies should be overpowered, and it should appear to be positive, in the presence of stronger negative groups. Thus, in phenol, the $\mathrm{C}_{6} \mathrm{H}_{5}$ group is negative by the above definition, since this compound has distinct acid properties. Hence, in this compound the $\mathrm{OH}$ is positive by contrast. Thus, phenol should act like aniline or toluene in the matter of relative ease of entrance of substituents; this it does.

To summarize, Pattern $0 . p$. rings may be produced by either "positive" or "negative" substituents, the determining factor being their net attractive force toward the adjacent aromatic electrons. Superimposed upon this is the general polarity of the ring whereby strongly negative substituents tend to prevent the release of the hydrogen in para and meta positions, while positive groups make it very easily substituted. The ease of substitution in ortho positions, however, is determined mainly by considerations of "steric hindrance" such as the local action of groups, rather than the general polarity of the ring.

In the case of Pattern $m$. rings the matter is considerably simpler, because it happens that all the substituents which form this pattern are strongly negative. Thus all Pattern $m$. rings should be more difficult to substitute than positive or weakly negative $o$. p. rings.

\section{Single-ring Aromatic Compounds Other Than Benzene}

Pyridine.-Pyridine ${ }^{9}$ (Fig. 6 ) is benzene with a nitrogen atom in place of a carbon-hydrogen. The aromatic electrons, in such a non-symmetrical

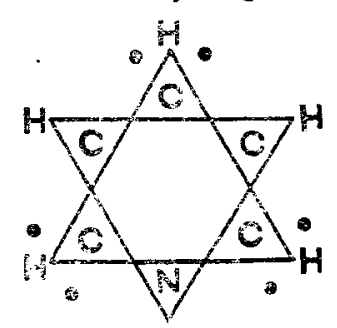

Fig. 6.-Pyridine: system, should take up Pattern $m$. arrangement, since there is no hydrogen over the nitrogen to attract them, making pyridine somewhat analogous to nitrobenzene. The substitution behavior is consistent with this assumption, since chlorine goes to the $\beta$ or meta position. The strongly "negative" substituents, $\mathrm{SO}_{3} \mathrm{H}$, and particularly $\mathrm{NO}_{2}$, have difficulty in substituting into this ring, probably due to the open meta positions being strongly negative in the case of pyridine sulfate and pyridine nitrate, because of the polar groups close to the nitrogen of the ring.

The open nitrogen atom, with a "free" pair of electrons, invites the addition of a hydrogen kernel, and a negative ion, to form a base or salt.

Pyridine, on treatment with hydrochloric acid, should be able to add the $\mathrm{H}$ as a positive ion with the formation of $\mathrm{C}_{5} \mathrm{H}_{5} \mathrm{NHCl}$. This is a wellknown compound, and is a typical result of the "basic" properties of

a Sidgwick, "Organic Chemistry of Nitrogen," Oxford, 1910. Brühl, "Chemie der Sechsgliedrigen Heterocyklische Systeme," 1898. 
pyridine. Water should and does also add to pyridine, to form the compound $\mathrm{C}_{5} \mathrm{H}_{5} \mathrm{NHOH}$.

Thiophene, Furane, and Pyrrol.--There are at least two possible arrangements of electrons which. will represent thiophene $\left(\mathrm{C}_{4} \mathrm{H}_{4} \mathrm{~S}\right)$ in this system. The one shown in Fig. 7 is that which is most nearly consistent with the arrangement in benzene, which thiophene closely resembles in physical and some chemical properties. It is similar to the type of "centric" formula postulated by Bamberger ${ }^{10} 30$ years ago to explain the aromatic behavior.

In this arrangement, the sulfur is "aromatic," with the two aromatic electrons it furnishes drawn closely to it, because of the 6 positive and only 2 negative charges which are near the aromatic electrons. This forces the 4 aromatic electrons furnished by the carbons to group around the $\beta$ carbons, and necessitates 2 electrons in the space between the $\beta$ carbone. The repulsion of these 2 electrons for each other means that they group very closely to the $\beta$ carbons. Sich an electronic grouping correspo:ads somewhat closely to Pattern o.p. arrangement of benzene, such as is indicated for phenol, but lacks the carbon in para position to the sulfur.

Substitution should be extremely easy into the $\alpha$ positions, those next to the sulfur-even easier than into phenol-on account of the

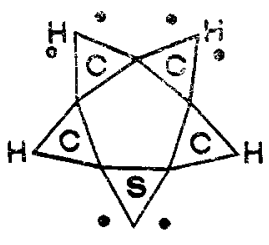

Fig. 7.

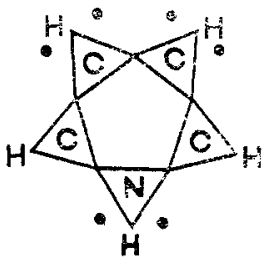

Fig. 8.

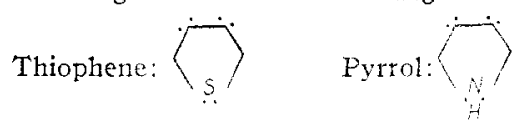
"openness" of the positions. Once these positions are filled, however, the electrons should then rearrange more into Pattern $m$. which leares the $\beta$ positions appreciably open for substitution.

The $\alpha$ positions of thiophene substitute with the greatest ease or even with violence. One of the $\beta$ positions substitutes fairly readily, once the $\alpha$ positions are filled, but the second $\beta$ position is only entered with considerable difficulty.

The formula of thiophene is much less symmetrical than that of benzene, only two planes of symmetry being possible and, as a consequence, the compound would be expected to be far less stable. If thiophene vapor is bubbled through well-cooled fuming nitric acid, a nitrothiophene can be obtained. However, ordinary treatment yields only oxidation products obtained from the broken ring. (Pyridine, while similarly unsymmetrical, is protected from easy decomposition by its larger size and its less reactivity.)

Furane $\left(\mathrm{C}_{4} \mathrm{H}_{4} \mathrm{O}\right)$ is of the same pattern as thiophene, with oxygen in ${ }^{10}$ Bamberger, Ber., 24, 1758 (1891). 
place of the sulfur. The oxygen octet is considerably smaller than that of sulfur, which brings the various atoms closer together around the ring, resulting in still greater tendency of the ring to rupture, due to internal repulsion. This compound is so easily destroyed that direct substitution behavior is completely masked.

Pyrrol $\left(\mathrm{C}_{4} \mathrm{H}_{4} \mathrm{HH}\right)$ is a 5 -membered ring ${ }^{11}$ with the radical $\mathrm{NH}$ in place of the sulfur of thiophene as shown in Fig. 8. The electron pattern should be like that of thiophene, with the electrons forcing each other apart in the space between the $\beta$ carbons. This Pattern $o . p$. arrangement of electrons leaves the $\alpha$ carbons wide-open, as are the ortho carbons of phenol. This causes pyrrol to resemble phenol closely in such reactions as involve a replaceable hydrogen and "open" adjacent positions. Even greater ease of substitution than in phenol, and the same ease of substitution $^{12}$ first to $\alpha$ and then to $\beta$ positions, as is the case with thiophene, should be and is the case here also. (The substitution is so easy that chlorine or bromine must be highly diluted to avoid over-violence of reaction and destruction of the ring. "Coupling" with diazonium compounds is likewise extremely easy.)

The pyrrol ring should be under the same degree of outward-acting strain as the furane ring, since the oxygen and nitrogen octets are of the same size. Like the furane ring, the pyrrol ring should tend to rupture. It is recorded that the pyrrol ring "expands" into the pyridine ring by losing the hydrogen of the nitrogen atom and adding a $\mathrm{CH}$ group. This expansion is to be expected as the result of rupture under the conditions when a $\mathrm{CH}$ group is available for addition. Such expansion cannot occur in the case of thiophene or furane, as the sulfur or oxygen cannot dispose of an electron as the $\mathrm{NH}$ group can, to become $\mathrm{N}$, and thus form a stable aromatic ring.

Conditions for Aromatic Structure. - In the light of the theory as above illustrated, it is interesting to see what appear to be the conditions which make possible the formation of the rather unique "aromatic" rings.

In the derivation of the new octet benzene ring, it was convenient to start with the Baeyer "centric" formula, particularly as the octet ring turns out to be more or less an interpretation of the "centric" formula. If, instead, the Kekule formula were the starting point, the same final result would have been attained. In this case, however, the cause considered for the formation of a 6-membered ring would have been the large repulsive force between the electrons at the middle points of the 3 -sided Kekule ring (see Fig. 1).

In the case of cyclo-octatetrene $\left(\mathrm{C}_{8} \mathrm{H}_{8}\right)$, there would be so little force tending to round out the square figure into an octagon that the ordinary

${ }^{11}$ Ciamician, Ber., 37, 4200 (1904).

${ }^{12}$ Mazzaro and Borgo, Gazz. chim. ital., 35, 477 (1905). 
alternate single and double bond formula should be expected to exist. Actually, this compound ${ }^{13}$ is stated to be entirely aliphatic in type.

One condition for aromatic st:ucture, as seen from the above, is a ring with considerable outward-acting strain, so that double bonds are bent open outwardly and pairs of extra electrons are forced to the outside of the ring.

Rings of but 3 or 4 members, such as $\mathrm{C}_{2} \mathrm{H}_{2} \mathrm{NH}, \mathrm{C}_{2} \mathrm{H}_{2} \mathrm{O}, \mathrm{C}_{4} \mathrm{H}_{4}, \mathrm{C}_{3} \mathrm{H}_{3} \mathrm{~N}$, etc., answer the above requirement for aromatic structure. (Groups such as $\mathrm{CH}$ or $\mathrm{N}$ would furnish one aromatic electron each, whereas $\mathrm{NH}$, $\mathrm{S}$ or $\mathrm{O}$ would furnish two each.) In these cases, however, and with any other 3- or 4 -member rings, there would be powerful disruptive forces, due to the atomic kernels repelling each other, at fairly close range. For this reason, stable aromatic compounds with 3 - or 4 -member rings are scarcely to be expected.

Five- and 6-member rings offer the most possibilities, many of which have already been considered. It is interesting to note that, in addition to the foregoing requirements, aromatic structure is observed only in those combinations of elements which furnish six extra or aromatic electrons above those needed to complete a single-bonded ring.

\section{Summary}

1. The octet or Lewis-Langmuir theory is considered as undergoing evolution, in that the original cubical octet has given way in certain cases to that of an octet which consists of 4 pairs of electrons arranged as are the points of a tetrahedron.

2. The pairing tendency of electrons is considered as possibly due to the presence of magnetic properties in the electrons (magnetons).

3. The Kekulé, Dewar and centric benzene formulas are expressed in octet nomenclature. They are considered as unstable forms.

4. A stable arrangement is worked out, and shown to be applicable not only to benzene, but to other aromatic compounds.

5 . In this arrangement there is a ring of 6 carbons, each single-bonded to its neighbor on either side, and to hydrogen. The remaining 6 electrons are placed between the carbons in the plane of the ring, thus forming an octet for each carbon atom.

6. These 6 "aromatic" electrons are considered as being in stable equilibrium only when paired up, near Carbons 1,3 and 5 , or 2, 4, and 6 , (or oscillating between these forms in benzene itself or in derivatives containing 6 identical substituents),

7. Substituents on the carbons are considered as favoring the retention of one or other grouping of electrons, according to their electrical action on the nearby electrons, resulting in Patterns $0 . p$. and $m$. Substituents ${ }^{13}$ Willstaetter and Waser, Ber., 44, 3423 (1911); C. A., 6, 748 (1912). 
with more than 5 kernel charges on the octet joining the ring plus its attached hydrogens, are indicated as favoring Pattern $o . p$. (in toluene and chlorobenzene), and those with 5 or less charges, as favoring Pattern $m$. (in nitrobenzene and benzaldehyde).

8. Substitution is considered as possible only when the hydrogens are lightly held ("open" positions of the adjacent aromatic electrons). The influence of these electrons is to "open" or "close" positions according to their attractive action on the hydrogen kernels.

9. Relative ease of substitution into the various substituted benzenes is treated as a result of polarity considerations, which are developed as an essential consequence of the octet theory, and an addition to the above aromatic structural considerations. Preference for substitution in ortho vs. para positions is treated in like manner.

10. Pyridine, thiophene, furane and pyrrol are treated similarly and afford striking confirmations of the postulated structure in their behavior.

11. Addition of any kind is shown to be inconsistent with the retention of aromatic structure in the remainder of the ring.

The writer wishes most heartily to thank the various members of the Research Laboratory of Applied Chemistry for their encouragement and assistance. In particular, he feels indebted to Professor Robert E. Wilson, the Director, for the large amount of time and thought which he gave most generously. The kind interest of Professors J. F. Norris and A. A. Blanchard is likewise much appreciated.

Cambridge 39, Massachusetts

[CONTRIBUTION FROM THE CHEMICAL LABORATORY OF THE UNIVERSITY OF ILLINOIS]

\section{THE IONIZATION CONSTANT OF HYPOCHLOROUS ACID.} EVIDENCE FOR AMPHOTERIC IONIZATION

\section{By William A. Noyes and Thomas A. Wilson}

Received March 16, 1922

In a very careful and extended experimental study published in 1899, Jakowkin ${ }^{1}$ demonstrated conclusively that chlorine is hydrolyzed by water to hydrochloric and hypochlorots acids. He determined the partition coefficient of chlorine between carbon tetrachloride and pure water and also between carbon tetrachloride and solutions of strong and weak acids, chlorides, and hypochlorous acid. He also determined the conductivity of pure hypochlorous acid solutions and of solutions of chlorine in water, and observed that the conductivity of the latter is almost wholly due to the hydrochloric acid formed by the hydrolysis, and that the hypochlorous acid is ionized scarcely at all under these conditions. Finally, he showed that hypochlorous acid volatilized more rapidly than hydrochloric acid

1 Jakowkin, Z. physik. Chem., 29, 613 (1899). 